NASA Contractor Report 198282

ICASE Report No. 96-11

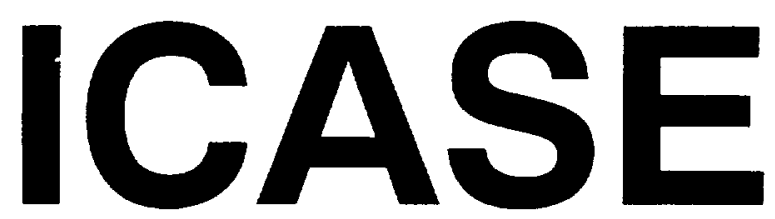

\title{
RESPONSE OF A PANEL STRUCTURE FORCED BY THE NOISE FROM A NEARLY SONIC JET
}

C. C. Fenno, Jr.
A. Bayliss
L. Maestrello

NASA Contract No. NAS1-19480

February 1996

Institute for Computer Applications in Science and Engineering

NASA Langley Research Center

Hampton, VA 23681-0001

Operated by Universities Space Research Association

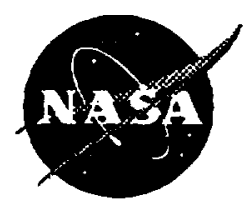

National Aeronautics and Space Administration

Langley Research Center

Hampton, Virginia 23681-0001 



\title{
Response of a Panel Structure Forced by the Noise From a Nearly Sonic Jet
}

\author{
C. C. Fenno, Jr.* \\ Research Associate \\ National Research Council, Hampton, VA 23681-0001
}

A. Bayliss ${ }^{\dagger}$

Professor, Department of Engineering Sciences and Applied Mathematics

Northwestern University, Evanston, IL 60208

\author{
L. Maestrello \\ Senior Staff Scientist \\ NASA Langley Research Center, Hampton, VA 23681-0001
}

\begin{abstract}
A model of a high subsonic jet with a nearby array of flexible, aircraft-type panels is studied numerically in two dimensions. The jet is excited by a limited duration, spatially localized starter pulse in the potential core. The long time evolution of unsteady disturbances in the jet, the responses of the panels and the ensuing radiation are computed. The results show that the spectral response of both the jet and the panels is concentrated in a relatively narrow frequency band centered at a Strouhal number (based on jet exit velocity) of approximately 0.25 and associated harmonics. The loading on the panels generally increases with downstream distance. Panel radiation is weakest in upstream directions. Interior zones of silence, due to destructive interference of radiation from the panels, are observed.
\end{abstract}

${ }^{*}$ The first author was supported by NASA Langley Research Center while in residence under a National Research Council Postdoctoral Research Associateship Award.

${ }^{\dagger}$ The second author was partially supported by the National Aeronautics and Space Administration under NASA Contract No. NAS1-19480 while in residence at the Institute for Computer Applications in Science and Engineering (ICASE), NASA Langley Research Center, Hampton, VA 23681-0001. Additional support was provided by NSF grant DMS 93-01635. 



\section{Introduction}

This paper describes the results of a numerical simulation of jet noise in the presence of four flexible aircraft-type panels in a panel-stringer assembly. The simulation is based on a model which fully couples the fluid dynamics of the jet flow to the panel motion and the resulting acoustic radiation. The primary objective is to determine the role played by near sonic jet exit conditions on installation effects from the nearby flexible structure and on the response and the acoustic radiation from the structure.

In this paper we consider the long time evolution of unsteady disturbances in a nearly sonic jet exiting from a converging nozzle together with panel responses and ensuing radiation from the panels. In previous work, jet acoustics, panel response and radiation have been considered for lower speed jets exiting from straight pipes, both at rest [1] and in forward motion [2]. The present work focuses on jet evolution over much longer timescales, emphasizing in particular vortex shedding from the nozzle lip.

In previous analyses, the exact sources of jet noise have been identified from the basic equations of fluid dynamics $[3,4,5,6]$. Generally, these exact sources have to be modeled in some way for computation. An important feature of the present method is the direct computation of at least some of the natural sources of jet noise, namely fluid dynamical instability waves which develop due to instability of the jet shear layer. Experiments have demonstrated the existence of large scale structures or instability waves in jets $[7,8,9]$. These structures are believed to act as sources of sound, a point also confirmed by analytical studies $[10,11,12,13]$ and computations $[9,14,15]$.

The geometry of the computational model is shown in figure 1. The Euler equations are solved in two domains; the jet domain and the radiation domain separated by an array of four flexible panels. Panel response and radiation are also computed and are fully coupled to the fluid dynamics in the sense that at each timestep the fluid dynamics (Euler) computation provides the pressure difference across the panels, thereby allowing computation of panel displacement and velocity. The resulting panel velocity then serves as a boundary condition for the Euler computation. Thus no modeling of the panel excitation is needed. Other approaches to the general problem of describing panel loadings are possible, e.g., [16]. 
The paper is organized as follows. In the next section there is a description of the model and a discussion of the numerical method and boundary conditions. Following that the results are presented first for the case of a free jet and then for the full jet flow/acoustic/structural interaction. Finally, the results are summarized and conclusions are drawn.

\section{Numerical Method}

The computational domain is shown in figure 1. Unsteady pressure, density and velocity are computed in two regions. The lower domain in figure 1 contains the jet, exiting from a contracting nozzle of width $D$, while in the upper domain, (modeling the aircraft interior) only disturbances due to the panel radiation are present. These two regions will be referred to as the jet and radiation domains, respectively. The wall boundary is a rigid wall containing four adjacent flexible panels (denoted as panels 1-4 in figure 1) with rigidly clamped boundaries. The panels vibrate in response to excitation by sound from the jet, thereby radiating acoustic disturbances into both domains. In the jet domain, the panel radiation level is significantly smaller than the acoustic disturbances generated in the jet, in contrast to the radiation domain where panel radiation is the sole source of acoustic disturbances.

The numerical method involves coupling the computation of a nonlinear equation governing the panel responses (the beam equation) to an Euler computation performed in both the jet and radiation domains. The panel vibration is fully coupled to the fluid dynamics in that at each time step the pressure difference across the panels, computed from the Euler computations, serves as a forcing term for the beam equation. Similarly, the displacement obtained from the beam equation is differentiated in time and is imposed as a boundary condition for the Euler computations. The numerical method has been described in detail [1]. Thus, the presentation here will be brief.

The nonlinear beam equation is

$$
D_{b} \frac{\partial^{4} z}{\partial x^{4}}-N_{x}(z) \frac{\partial^{2} z}{\partial x^{2}}+\rho_{b} h \frac{\partial^{2} z}{\partial t^{2}}+\gamma \frac{\partial z}{\partial t}=p^{+}-p^{-}
$$

where $z$ represents the beam transverse deflection, $\rho_{b}$ is the mass per unit volume of the beam, $h$ is the beam thickness, $\gamma$ is the physical damping, $D_{b}=M h^{3} / 12\left(1-\nu^{2}\right)$ is the 
stiffness of the beam, $M$ is the modulus of elasticity and $\nu$ is the Poisson ratio of the beam material. The coefficient $N_{x}(z)$ of the nonlinear term represents the tension created by the stretching of the plate due to bending. The pressures in the radiation and jet domains are $p^{+}$and $p^{-}$, respectively. The solution of equation (1) is obtained at each time step using an implicit finite difference method. The panels are assumed clamped at both ends.

The coupling of the beam computation to the Euler computation occurs through the forcing term given on the right hand side of equation (1). The pressures $p^{+}$and $p^{-}$are obtained from the Euler computation using an explicit scheme. The displacement at the new time level is then obtained from solving equation (1) one time step. The normal velocity, $v$, is then obtained from differentiating $z$ and employed as a boundary condition to complete the update to the Euler computation. Since this procedure is employed at each time step, the fluid and structural calculations are fully coupled.

The Euler equations are solved in conservation form for the vector

$$
\hat{\mathbf{w}}=(\rho, \rho u, \rho v, E)^{T}
$$

where $\rho$ is the density, $u, v$ are the $x$ and $y$ components of the velocity respectively and $E$ is the total energy per unit volume,

$$
E=\frac{1}{2} \rho\left(u^{2}+v^{2}\right)+c_{v} \rho \tilde{T}
$$

where $\tilde{T}$ is the temperature and $c_{v}$ is the specific heat per unit volume. The pressure, $p$, is obtained from the equation of state. The Euler equations are solved separately in both the jet and radiation domains.

In the jet domain the Euler equations are modified to account for the jet flow. The jet exits from a nozzle of width $D$ and the solution is computed both within and exterior to the nozzle. The Euler equations are modified to account for two different non-homogeneous forcing terms [1]. One term serves as a starter pulse to excite the jet. It corresponds to a localized source of mass injection at the location $\left(x_{s}, y_{j}\right)$, where $y_{j}$ is the location of the jet axis (3.5D from the wall) and $x_{s}$ is approximately $1.15 D$. An alternative approach, involving time harmonic excitation of the jet is described in [17]. The second forcing term is designed so that in the absence of the starter pulse the solution to the Euler equations would be 
a stationary profile corresponding to a spreading jet. The inclusion of this term separates the computation of the disturbance, in particular the resulting instability waves, from the computation of the mean flow (i.e. the spreading jet). Thus, the resulting system of equations allows for the simulation of instability waves and the resulting sound generation, together with the curving of acoustic waves in the jet flow field, without requiring the computation of the spreading jet itself. Although this is a simplified model, it captures many of the observed features of instability wave generated jet sound and permits high resolution computation of the coupling of jet noise with the flexible panels and the resulting radiation from the panels. In particular, the model allows for computation of the natural sources of jet noise (the instability waves) together with the sound radiated by these sources.

The initial conditions are taken to be ambient data in the radiation domain and the mean state in the jet domain. The model for the mean axial and normal velocities is obtained from [18] in Cartesian coordinates. In contrast to previous calculations [1, 2] at lower jet speeds, the current study requires a mean flow model which addresses the compressibility of the flow. Thus, the mean pressure and density are also modeled. Inside the nozzle, the quasi 1-D equations of isentropic flow are employed for pressure and temperature. Outside the nozzle, the jet pressure is obtained from [19]. Centerline temperature is assumed isentropic and off-centerline temperature variations follow the axial velocity relaxation. Density follows from the state equation both inside and outside the nozzle. It is imperative that the mean flow be smooth up to and including its second derivative for the numerical simulation of instability flows [20,21]. Therefore, great care was taken in modeling the mean flow.

The boundary conditions are as follows (refer to figure 1):

1. Bounding wall - rigid conditions are imposed except for the flexible panels which are treated as described above.

2. Nozzle exterior - impedance boundary conditions are used on the exterior of the nozzle, simulating the use of an absorbing material to absorb waves incident on the nozzle from the exterior.

3. Nozzle interior - rigid conditions are used for the terminal two thirds of the nozzle. The first third (near the inflow boundary) employs impedance conditions to reduce 
the possibility of reflections from the nozzle inflow.

4. Inflow for the nozzle - characteristic conditions. Specifically, the Euler equations are linearized about the ambient state, assumed to hold far upstream in the nozzle, and the three incoming characteristics are imposed.

$$
p+\rho c u, \quad v, \quad c_{\infty} \rho-p / c_{\infty}
$$

to be the values that they would have far upstream. It has been shown that this boundary condition is valid for the lowest propagating mode in the nozzle [9].

5. All other boundaries in the problem are artificial. Non-reflecting (radiation) boundary conditions are imposed to prevent spurious reflections from propagating into the interior. These boundary conditions are based on a far field expansion of the solution $[22,23]$.

A finite difference scheme is employed which is fourth order accurate in space and second order in time. The scheme is a generalization of the second order MacCormack scheme to allow higher order accuracy in space [24]. The scheme is discussed in detail in $[9,15]$.

\section{Results}

The results are presented in two major sections; (i) simulation of a jet without a nearby structure (i.e. free jet) and (ii) the jet with the nearby structure. In each case, the jet exit Mach number is $M_{j}=0.9$ and the surrounding fluid is at rest. The nozzle utilizes a converging geometry with a contraction ratio of $2: 1$ over a longitudinal distance of four exit widths. Figure 1 shows a sketch of the computational domain for the case which includes the structure.

The grids used in this study utilize stretching to enhance resolution in $y$ of the shear layer and resolution in $x$ near the nozzle exit. The $y$-grid is mapped to conform to the contracting nozzle, while the $x$-grid remains Cartesian. This deviates slightly from orthogonality in the vicinity of the nozzle contraction but returns to a standard stretched Cartesian grid in all other regions. The computations have been extensively validated by grid refinement $[1,2]$ and, equally important, there are no spurious boundary reflections visible for the time interval considered in this paper $\left(t c_{\infty} / D \leq 100\right)$. 


\subsection{Free Jet Results}

For this case, non-reflecting boundary conditions are imposed on the upper boundary and the jet is centered in $y$. The origin of the $x$-coordinate is at the nozzle exit. The domain extends $45 \mathrm{D}$ downstream, $30 \mathrm{D}$ upstream and $30 \mathrm{D}$ in both directions normal to the jet axis. The computed solution is symmetric around the jet axis to a close approximation, although symmetry is not imposed in the computation. A $581 \times 901$ grid is employed.

The structure of the pressure and vorticity field near the nozzle exit are considered first. Shown in figure 2 are contours of the instantaneous vorticity and pressure fluctuation,

$$
\tilde{p}(x, y, t)=p(x, y, t)-\bar{p}(x, y)
$$

where $\bar{p}$ denotes the mean pressure, for a small region near the nozzle exit at $t c_{\infty} / D=100$. The sources simulated by the model are vorticity disturbances shed from the nozzle lip due to the near discontinuity in the velocity profile at the lip. The shedding is nearly periodic and the vortices convect primarily downstream. As they propagate, pressure disturbances associated with the vortices travel both upstream and downstream, ultimately leading to far field sound. The effect of the flow field inside the nozzle on the wavelength of the upstream propagating disturbances can be seen from the shorter wavelengths due to the slower upstream wave speed in the contracted section.

Figure 3 shows the time history, spectrum and phase plot of the fluctuating pressure, $\tilde{p}$, at a point inside the jet flow field $\left(x=10 D, y-y_{a x i s}=0.8 D\right)$. The PSD is normalized to $0 \mathrm{db}$ based on the maximum of the spectra. The figure shows that the spectral structure of the flow field $\tilde{p}$ is composed of a fundamental frequency, $f^{*}$, such that the Strouhal number, $S_{t}^{*} \equiv f^{*} c_{\infty} / D \simeq 0.25$. The spectrum also shows two harmonics and one quarter subharmonic. This fundamental Strouhal number is close to observations of peak jet output (e.g., [25]). The precise value of $S_{t}^{*}$ is certainly a property of the particular model used in the computations. However, this scaling for the shedding frequency has been confirmed for the model by computations with different jet Mach numbers in a limited range around 0.9 and with different jet widths. Examination of $\tilde{p}$ over an earlier time window (not shown) indicates a more broadband structure due to sound generated from the leading instability wave generated from the starter pulse. The predominant long time feature of the excited 
jet predicted by the model is the nearly periodic shedding of vorticity from the nozzle lip. Computations with lower speed jets (not shown) indicate that this behavior becomes much more pronounced as the jet approaches a sonic exit velocity.

The unsteady flow field in the jet generates acoustic disturbances which propagate into the far field as sound. Figure 4 shows a color representation of $\tilde{p}$ over the entire computational domain at $t c_{\infty} / D=100$. Although the flow is initially excited by a starter pulse, at the time for the figure this initial disturbance has propagated through and exited the computational domain. The remaining disturbances are generated due to instabilities of the jet shear layer. These disturbances convect downstream and spread with the jet. Analogous structures have been previously observed and computed in axisymmetric models [7, 9]. The figure also shows a series of waves propagating upstream into the far field together with a similar series of waves propagating downstream into the far field. The waves appear to be nearly periodic and are of similar spatial extent. Furthermore, they have the same frequency and wavelength. These waves are due to the nearly periodic shedding of vorticity from the nozzle lip (see figure 2) and the subsequent generation of pressure disturbances both inside and outside the jet. It can be seen from figure 4 that the upstream propagating waves appear to emanate from a point closer to the nozzle exit than the downstream propagating waves and also appear to be out of phase with the downstream propagating waves. This results in a region of interference between these two wave patterns along a ray directed approximately $100^{\circ}$ from the jet axis.

There is also a wave pattern within the contracting nozzle. The fluid field within the nozzle is nearly constant in $y$, leading to a pattern of nearly plane waves. The boundary condition inside the nozzle is rigid up to $x=-10 D$ and then is gradually changed to an absorbing boundary condition to help eliminate reflections from the inflow boundary. Animation of this data indicates that only upstream propagating waves are present in the nozzle.

The far field pressure fluctuation is plotted in figure 5 as a function of $t c_{\infty} / D$ at three far field angles $\left(\theta=30^{\circ}, 90^{\circ}\right.$ and $\left.120^{\circ}\right)$ taken on a circle of radius $20 \mathrm{D}$. At all three points selected, $\tilde{p}$ oscillates with a nearly constant period with a gradually varying amplitude. The period is close to that of the vorticity generation from the nozzle lip. This phenomenon is transmitted to the far field as sound.

In addition to the time history, the PSD of $\tilde{p}$ and phase plots are shown in figure 5 . 
The PSD is normalized to $0 \mathrm{db}$ based on the maximum of the spectra over the three angles. The figure shows that the far field spectral structure is similar to the jet flow field spectra (figure 3). The fundamental frequency corresponds to $S_{t}^{*} \simeq 0.25$ and the two harmonics and one quarter subharmonic are exhibited as in the jet flow. The amplitude of the first and second harmonics is increased, relative to the fundamental, if the amplitude of the starter pulse is increased, confirming that these harmonics are generated via nonlinear interactions. Furthermore, the presence of low level subharmonics in the pressure spectra, again indicative of weak nonlinearities, are seen. Finally, it is seen that the spectra reach a maximum at $\theta \simeq 30^{\circ}$, consistent with experiments and previous computations $[2,8,9]$. The relative harmonic content of the time signal increases as $\theta$ increases. Thus there is a preferred upstream directivity for high frequencies (i.e. the harmonics).

\subsection{Jet/Structure Interaction}

Referring again to Figure 1, a wall, assumed infinite in extent and parallel to the nozzle, is located $3.5 \mathrm{D}$ above the jet. The wall is assumed rigid, except for four flexible, aluminum, aircraft-type panels with clamped boundaries. Each of the panels is of length $3.5 \mathrm{D}$ and thickness $0.01 D$. The panels are centered at $x / D=0,3.75,7.50$, and 11.25 , respectively. These are referred to as panels $1,2,3$ and 4 . Characteristic parameters of the panels are typical of aluminum.

The grid in the jet domain is comparable to that described above for the free jet. In order to allow a greater distance between the jet and the artificial lower boundary the $y$ domain extends down $45 \mathrm{D}$. The panels are at the origin in the vertical direction. The Euler equations are also solved in the radiation domain. Using the fully coupled model, the unsteady disturbances in the jet are computed, which serve to excite the panels, the responses of the panels, and the radiation from the panels into both the jet domain and the radiation domain.

A perspective plot of the unsteady flow and acoustic field can be seen in figure 6 . In the jet domain the large scale structure propagating downstream is observed, trailed by other pressure disturbances in the jet. The generation of disturbances from the nozzle lip is clearly visible and occurs on a continual basis as time evolves. There is a series of ripples corre- 
sponding to acoustic disturbances which radiate into the far field. The upstream radiation again appears to emanate from sources close to the nozzle exit, while the downstream radiation emanates from sources further downstream resulting in an interference pattern around $\theta=100^{\circ}$. The waves radiated downstream have higher amplitudes and the peak radiation occurs at around $30^{\circ}$.

A series of outwardly propagating ripples, representing acoustic radiation from the panels, can be seen in the radiation domain. Since these ripples emanate from all four panels it is not possible to ascribe a precise geometric origin to them. Thus, instead of angles, far field locations in the radiation domain are designated by $x / D$ values along a line at $y / D=25$. The wavelength is comparable to the wavelength of the downstream radiated sound in the jet, i.e. the wavelength and frequency is controlled by $S_{t}^{*}$. The wave pattern in the radiation domain is more sharply defined that that observed in [1] for a lower Mach number jet. The results in [1] concentrated on earlier times and slower jets than the present computation. The largest overall level of sound radiation occurs at roughly $90^{\circ}$ and the level is larger in downstream directions than in upstream directions.

Features of the fully coupled flow/acoustics/structural flow field are described next. The jet domain, panel response and radiation domain are examined.

\subsubsection{Jet Flow Domain}

In figure 7 the far field directivity is shown for both the free jet and the jet in the presence of a nearby wall. The data is given along an arc $20 \mathrm{D}$ from the jet exit and for angles below the centerline. Far field pressures with the wall are similar to those described previously for the jet without any wall. Over the time interval considered, the differences are small.

In figure 8 , the incident pressure at the center of the panels is shown in both the time and frequency domains. Phase plots are also shown. The overall level of $\tilde{p}$ increases with the downstream distance of the panels. The level of $\tilde{p}$ on panel 1 (partially upstream of the nozzle exit) is reduced nearly an order of magnitude from the loading on panel 4 . Since panel 4 is at a lower angle to the jet axis, this is in agreement with the directivity results in figure 7 . The frequency spectra of the incident pressure is dominated by the fundamental frequency, $S_{t}^{*}$, and its harmonics. The relative harmonic content of the incident $\tilde{p}$ for panel 1 
is significantly increased as compared to the other (more downstream) panels, due to the preferred upstream beaming of high frequencies.

\subsubsection{Panel Response}

The vibrating velocity, $v$, at the center of each panel is plotted in figure 9 , together with the corresponding PSDs and phase plots. The panels were held fixed until the leading pulse had passed. This eliminates any effect of the pulse in the panel response, but does lead to an impulsive start to the panel motion. For this reason, only the long-time response of the panels is investigated. The panel response increases with distance until reaching its maximum on panel 3 and then decreases slowly with distance. The relative subharmonic level is increased as compared to the incident pressure. This may be due to enhanced receptivity of the panels to low frequency excitation. The predominant response of the panels, however, is at the jet fundamental frequency, $S_{t}^{*}$, and its harmonics. The relatively large harmonic contribution to the response of panel 1 is indicative of the preferred upstream beaming of high frequencies, although the overall response is smaller than that of the more downstream panels.

The observation made in $[1,2]$ that the panels act as narrow filters, converting a broad band input into a narrow band response, may no longer be relevant for jets dominated by the long-time vortex shedding considered here. In this case the panels respond clearly at the frequencies of the loading, somewhat enhancing the subharmonic response.

\subsubsection{Radiation Flow Domain}

The panel radiation is considered next. The pressure, $\tilde{p}$, is computed along the line $y=25 D$ in the radiation domain. Shown in figure 10 are time histories, PSDs and phase plots for four different $x$-locations along this line. It can be seen from the figure that the overall level of radiation is reduced as $x$ decreases, i.e. in the upstream direction in terms of the jet flow field. The PSDs indicate that for downstream locations, there is peak radiation at $S_{t}^{*}$, the first two harmonics and the subharmonic. For upstream locations, a low level, relatively broad band spectrum is found. Thus the radiated sound is beamed primarily in vertical and downstream directions and is controlled by $S_{t}^{*}$, i.e. the vortex shedding frequency 
of the jet.

In figure 11 the overall intensity is computed as a function of $x$. This figure illustrates the strong vertical and downstream beaming of the panel radiation. It also illustrates a zone of relative silence at about $x / D=15$ where the intensity is reduced by about $15 \mathrm{db}$ from nearby locations. This zone can also be seen in the flow field in figure 6 and is due to destructive interference between radiation from the various panels. No such zone was observed at lower jet Mach numbers [1, 2], where the resulting radiation was more heavily influenced by the starter pulse and the leading instability wave generated sound in the jet.

In a real jet, instabilities can be generated by bursts within the jet, generating large scale structures, as simulated by the starter pulse and the intermediate time behavior of the jet. Also, instabilities can be generated in a more continual fashion via vortex shedding, as is

done here, by considering the long time evolution of the model. The model allows for a separation of these effects so that they can be studied separately.

\section{Conclusions}

A numerical simulation has been conducted of a nearly transonic jet exhausting into a fluid at rest both with and without the presence of a nearby structure. The structure is rigid except for four flexible aircraft-type panels. These panels respond to acoustic forcing from the jet and radiate sound into both the jet domain and a domain on the other side of the panels. The simulation utilized a full coupling between the Euler equations (solved on either side of the panels) and the nonlinear beam equation resulting in a full coupling between the flow, acoustics and panel response and radiation. The results are in qualitative agreement with experimentally observed phenomena and with previous computations indicating the present model is capable of capturing the important features of jet flow/acoustic/structural interactions at nearly sonic jet velocities.

In particular, the results indicate the following properties of the long time response of excited, high subsonic Mach number jets:

1. There is a nearly periodic shedding of vorticity from the nozzle lip. This shedding is associated with pressure disturbances which lead to far field sound. 
2. The shedding frequency scales with Strouhal number. $S_{t}^{*}$ is near 0.25 which is near the peak of many observed far field radiation patterns.

3. The relative harmonic content of $\tilde{p}$ increases with upstream angle.

4. The far field sound peaks near $30^{\circ}$.

All of the above are consistent with the features of previously observed real jets. Furthermore, the panel response and radiation results indicate that:

1. Loading and response of the panels generally increases with downstream distance.

2. The frequency spectrum of the panel responses is dominated by the jet shedding frequency, $S_{t}^{*}$, its harmonics and subharmonics.

3. The filtering action of the panels previously reported $[1,2]$ is less evident in the present work, where the panel forcing is dominated more by the periodic shedding from the nozzle lip and is already of a narrow band nature.

4. The relative level of harmonics in the panel loading and response is increased for upstream panels.

5. Radiation is primarily in vertical and downstream directions.

6. The upstream radiation is small compared to downstream radiation and does not appear to reflect the jet shedding frequency.

7. Zones of relative silence occur due to destructive interference.

Finally, the code developed is computationally efficient, running at over 190 MFLOPS on a Cray-YMP. This produces a complete solution of the fully coupled flow/acoustic/structural interactions in approximately $6 \mathrm{CPU}$ hours.

\section{Acknowledgments}

The authors thank Dr. J. C. Hardin for helpful discussions and comments. 


\section{References}

[1] McGreevy, J.L., Bayliss, A. and Maestrello, L., "Interaction of Jet Noise with a Nearby Panel Assembly," AIAA Journal, Vol. 33, 1995, pp. 577-585.

[2] Bayliss, A., Maestrello, L., McGreevy, J.L. and Fenno, C.C., "Response of Multi-Panel Assembly to Noise From A Jet In Forward Motion," AIAA Paper 95-0139, 1995.

[3] Lighthill, M.J., "On Sound Generated Aerodynamically-I, General Theory," Proceedings of the Royal Society, Vol. A222, 1954, pp. 1-32.

[4] Lilley, G.M., "Theory of Turbulence Generated Jet Noise: Generation of Sound in a Mixing Region," U.S. Air Force Technical Report AFAPL-TR-72-53, IV, Wright Patterson AFB, 1972.

[5] Ribner, H.S., "Dryden Lecture, Perspectives on Jet Noise," AIAA Journal, Vol. 19, 1981, pp. 1513-1526.

[6] Ribner, H.S., "An Extension of the Lighthill Theory of Jet Noise to Encompass Refraction and Shielding," NASA Technical Memorandum 110163, 1995.

[7] Crow, S. and Champagne, F., "Orderly Structure in Jet Turbulence," Journal of Fluid Mechanics, Vol. 48, 1971, pp. 457-591.

[8] Kibens, V., "Discrete Noise Spectrum Generated by an Acoustically Excited Jet," AIAA Journal, Vol. 18, 1980, pp. 434-441.

[9] Maestrello, L., Bayliss, A. and Turkel, E., "On the Interaction of a Sound Pulse with the Shear Layer of an Axisymmetric Jet," Journal of Sound and Vibration, Vol. 74, 1981, pp. 281-301.

[10] Bechert, D.W. and Pfizenmaier, E., "On the Amplification of Broadband Jet Noise by Pure Tone Excitation," Journal of Sound and Vibration, Vol. 43, 1975, pp. 581-587.

[11] Huerre, P. and Monkewitz, P.A., "Local and Global Instabilities in Spatially-Developing Flows," Annual Review of Fluid Mechanics, Vol. 22, 1990, pp. 473-537.

[12] Michalke, A. and Hermann, G., "On the Inviscid Instability of a Circular Jet With External Flow," Journal of Fluid Mechanics, Vol. 114, 1982, pp. 343-359. 
[13] Michalke, A., "Survey on Jet Instability Theory," Progress in Aerospace Science, Vol. 21, 1984, pp. 159-199.

[14] Bayliss, A., Maestrello, L. and Turkel, E., "On the Interaction of a Sound Pulse With the Shear Layer of an Axisymmetric Jet, III: Non-Linear Effects," Journal of Sound and Vibration, Vol. 107, 1986, pp. 167-175.

[15] Maestrello, L. and Bayliss, A., "Flowfield and Far Field Acoustic Amplification Properties of Heated and Unheated Jets," AIAA Journal, Vol. 20, 1982, pp. 1539-1546.

[16] Dowell, E.H., "Generalized Aerodynamic Forces on a Flexible Plate Undergoing Transient Motion in a Shear Flow with an Application to Panel Flutter," AIAA Journal, Vol. 9, 1971, pp. 834-841.

[17] Mankbadi, R., Hayder, M. and Povinelli, L., "The Structure of Supersonic Jet Flow and Its Radiated Sound," AIAA Journal, Vol. 32, 1994, pp. 897-906.

[18] Maestrello, L., "Acoustic Energy Flow From Subsonic Jets and Their Mean and Turbulent Flow Structure," Ph.D. Thesis, University of Southampton, England, UK, 1975.

[19] Maestrello, L. and McDaid, E., "Acoustic Characteristics of a High-Subsonic Jet," AIAA Paper 70-0234, 1970.

[20] Macaraeg, M.G., Streett, C.L. and Hussaini, M.Y., "A Spectral Collocation Solution to the Compressible Stability Eigenvalue Problem," NASA Technical Paper 2858, 1988.

[21] Fenno, C.C., Streett, C.L. and Hassan, H.A., "Use of Finite Volume Schemes for Transition Simulation," AIAA Journal, Vol. 30, 1992, pp. 1122-1125.

[22] Bayliss, A. and Turkel, E., "Radiation Boundary Conditions for Wave-Like Equations," Communications on Pure and Applied Mathematics, Vol. 33, 1980, pp. 707-725.

[23] Bayliss, A. and Turkel, E., "Far-Field Boundary Conditions For Compressible Flows," Journal of Computational Physics, Vol. 48, 1982, pp. 182-199.

[24] Gottlieb, D. and Turkel, E., "Dissipative Two-Four Methods For Time-Dependent Problems," Mathematics of Computation, Vol. 30, 1976, pp. 703-723.

[25] Zaman, K.B.M.Q. and Yu, J.C., "Power Spectral Density of Subsonic Jet Noise," Journal of Sound and Vibration, Vol. 98, No. 4, 1985, pp. 519-537. 


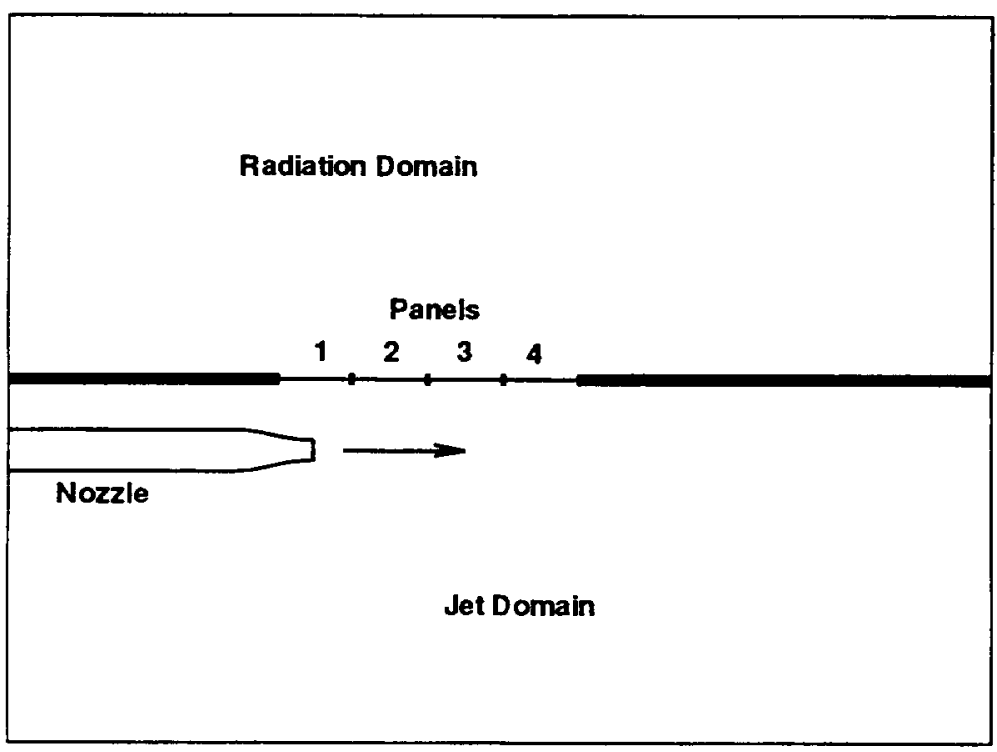

Figure 1: Computational Domain
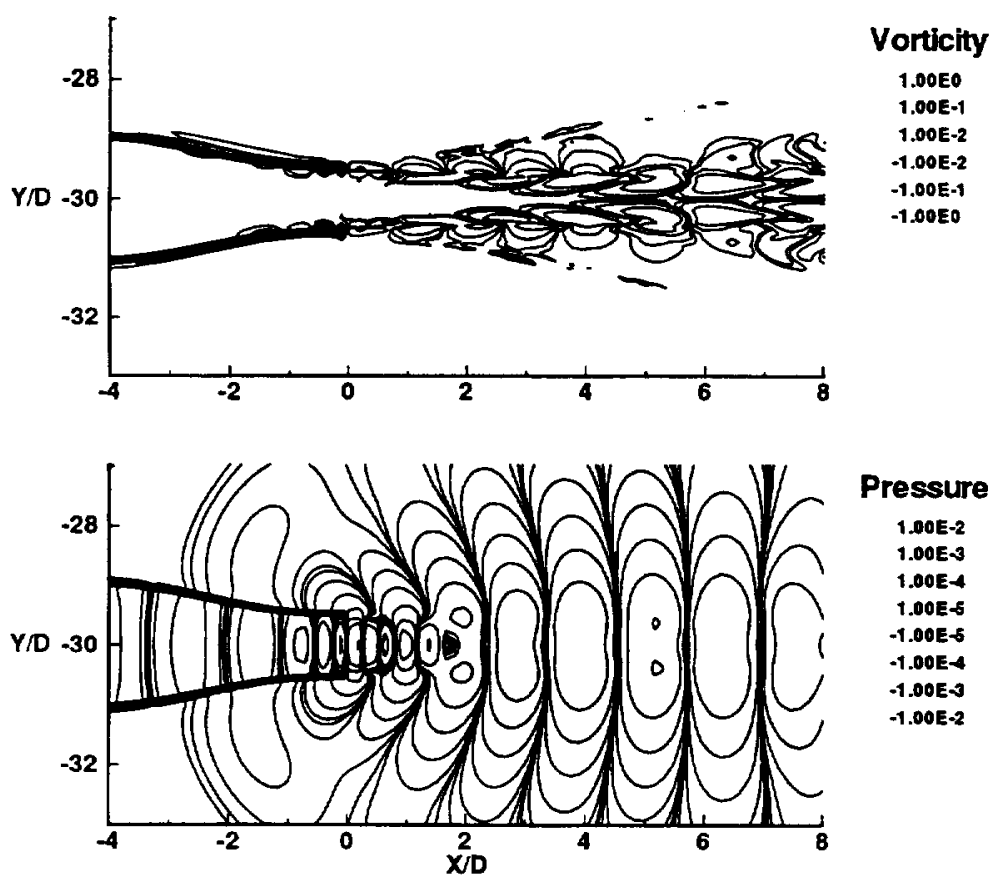

Figure 2: Near field vorticity and pressure contours 


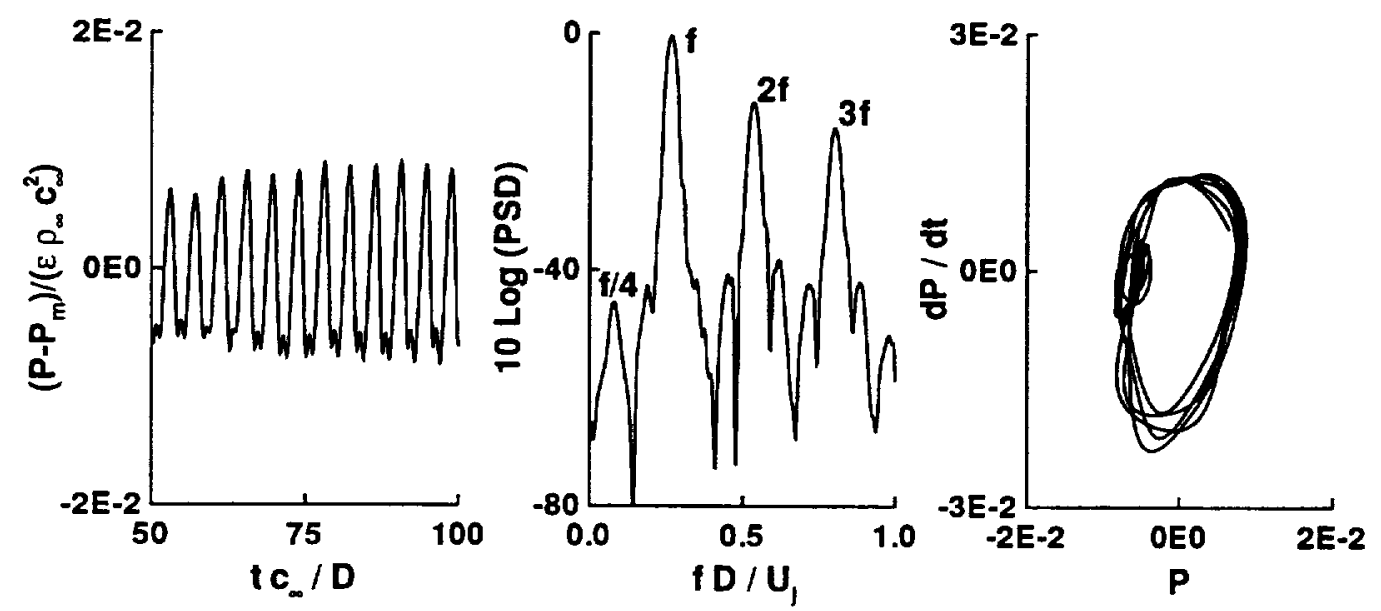

Figure 3: Jet flow field time history, spectrum and phase plot at $x / D=10$ and $\left(y-y_{a x i s}\right) / D=0.8$

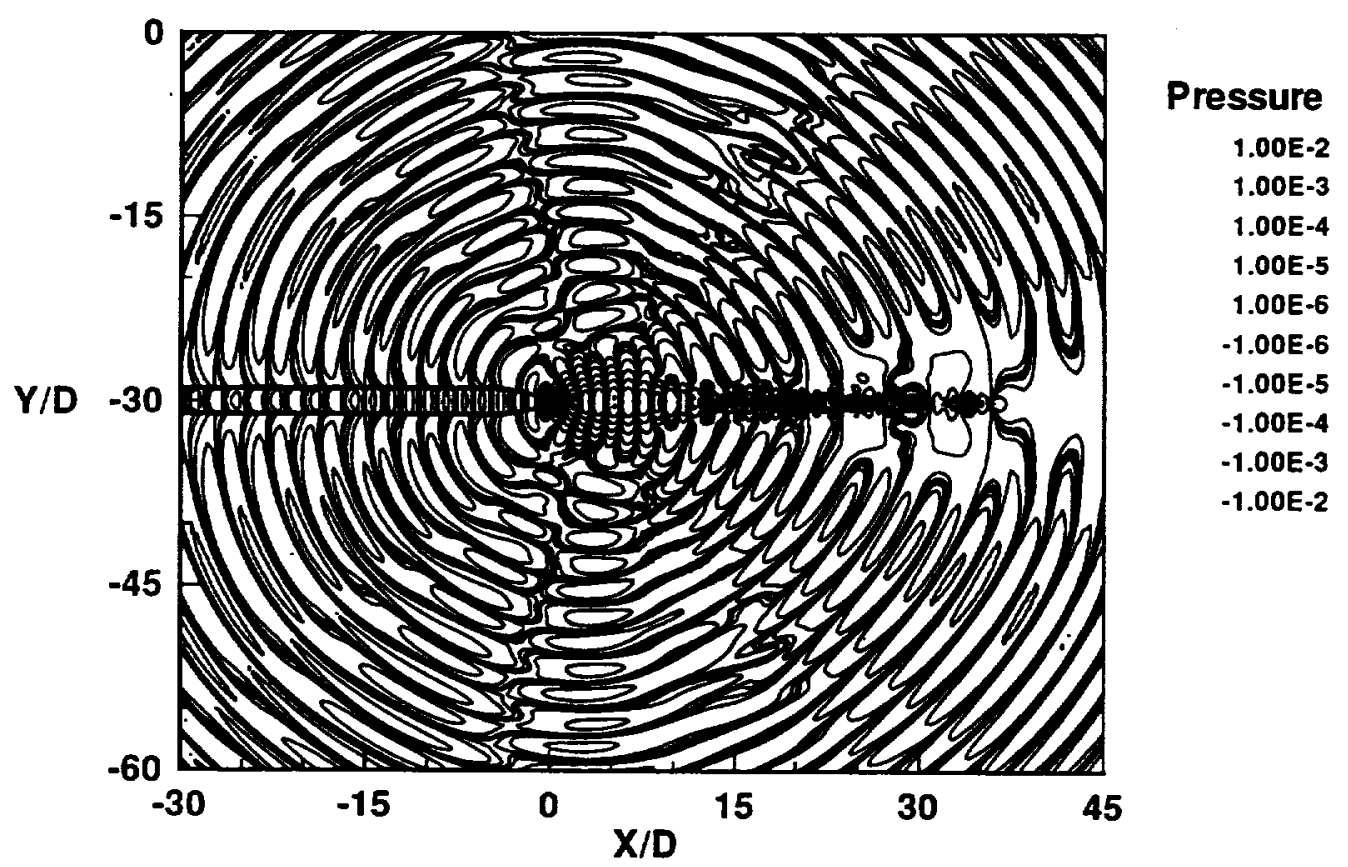

Figure 4: Contours of $\tilde{p}$ for free jet 

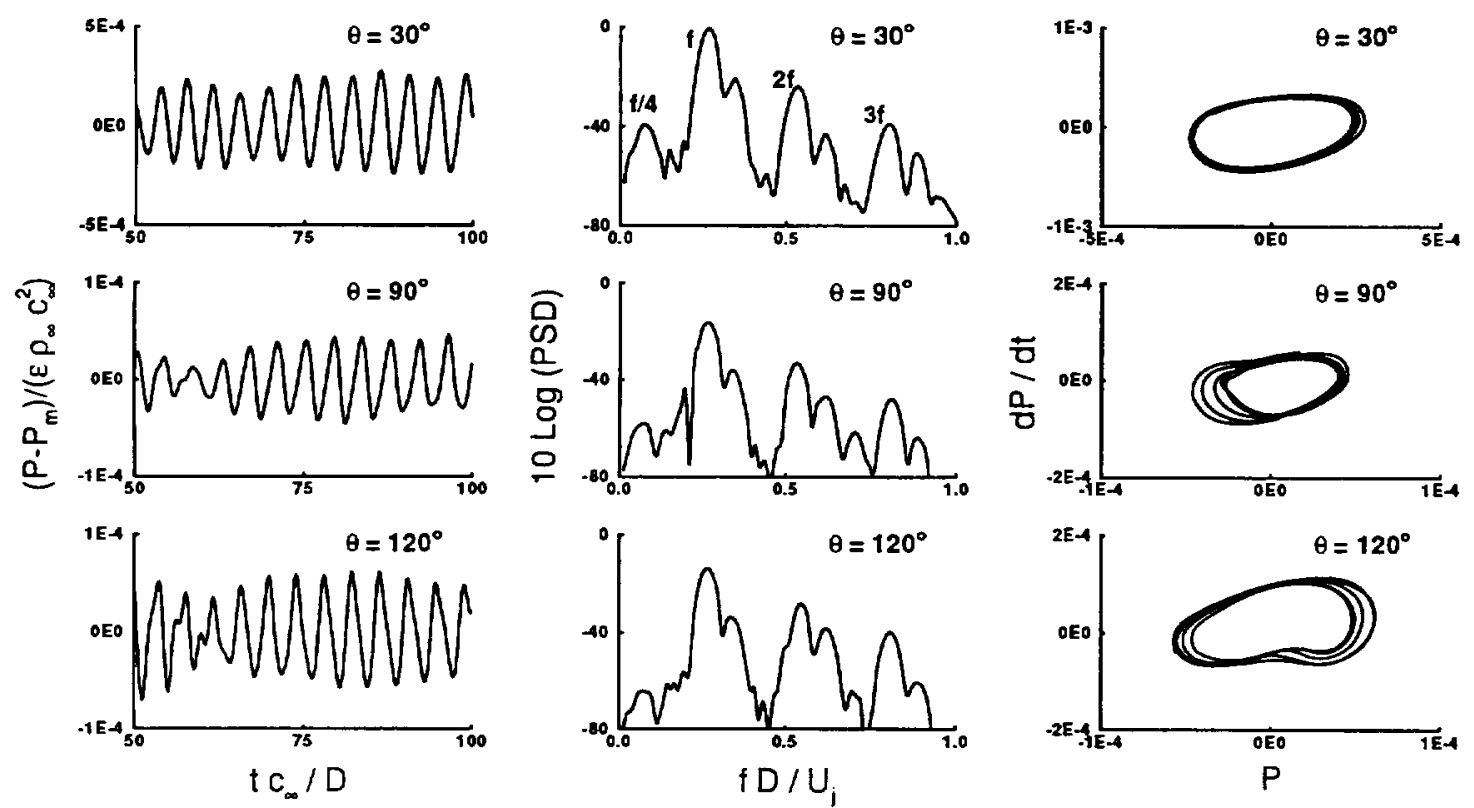

Figure 5: Jet far field time history, spectra and phase plots at $\theta=30^{\circ}, 90^{\circ}$ and $120^{\circ}$ from the jet axis

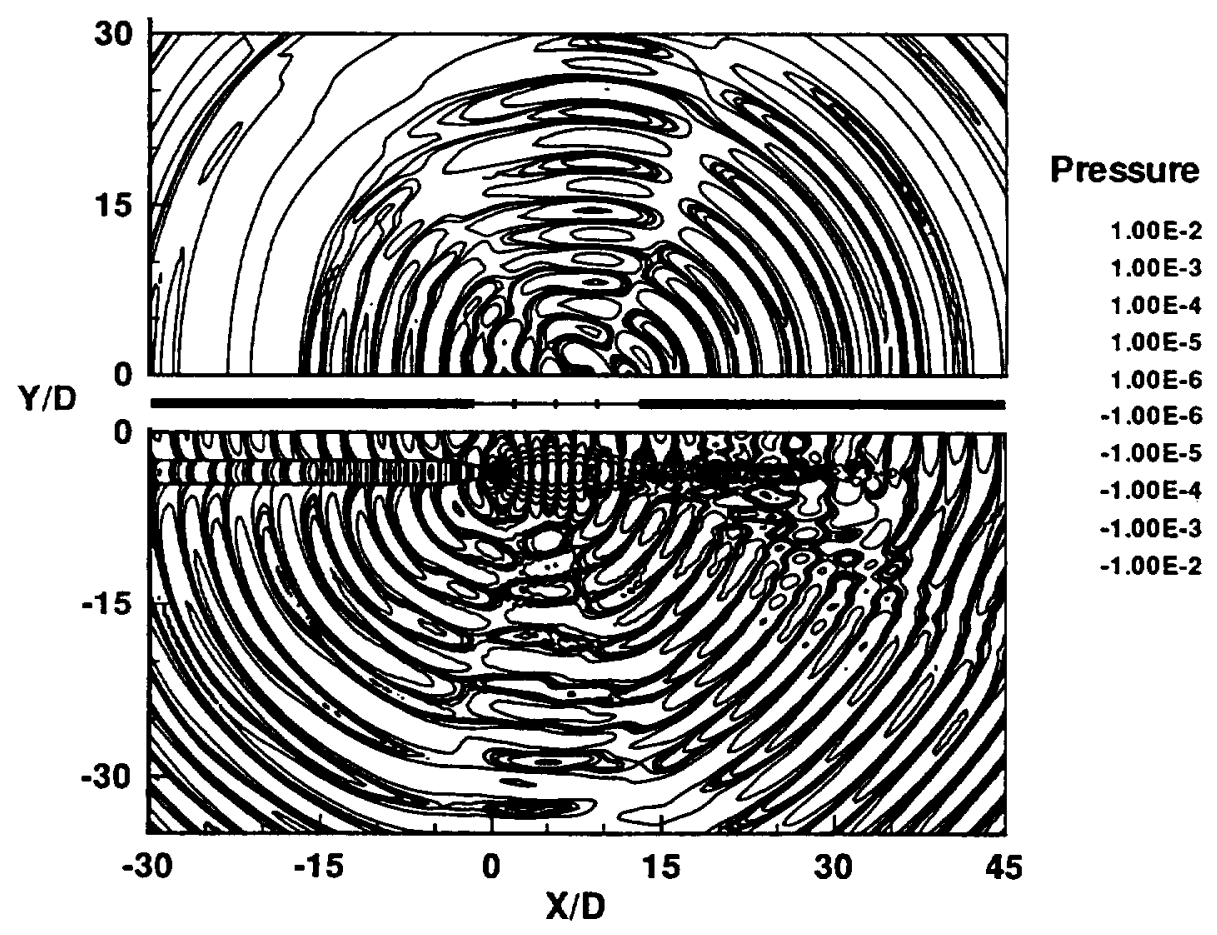

Figure 6: Contours of $\tilde{p}$ in jet and radiation domains 


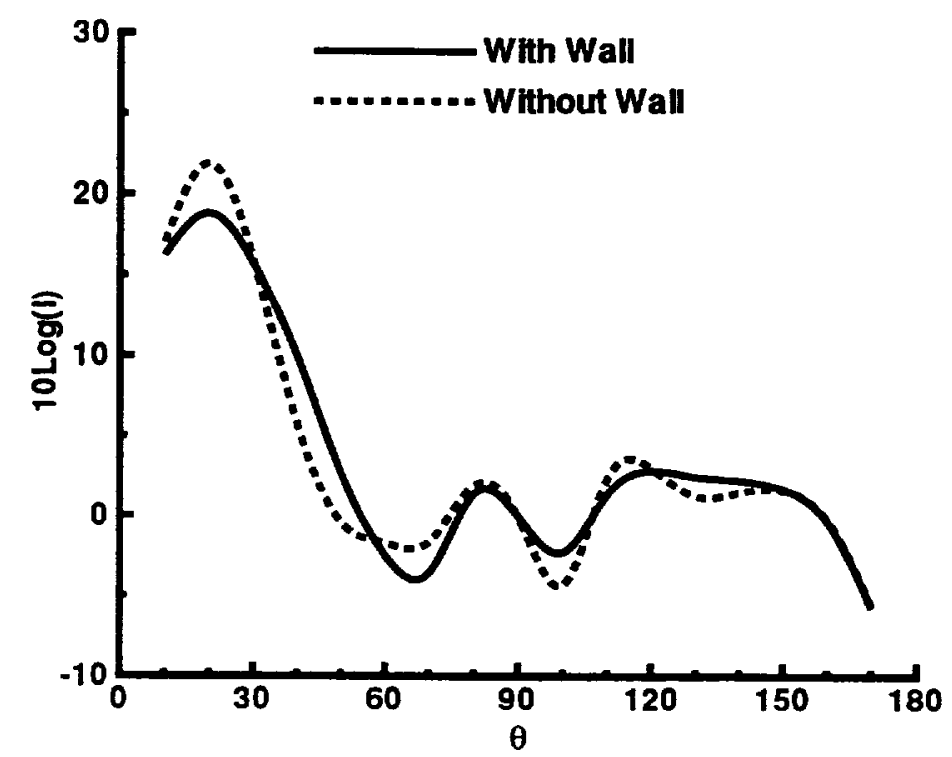

Figure 7: Jet directivity of overall sound pressure level, with and without nearby structure
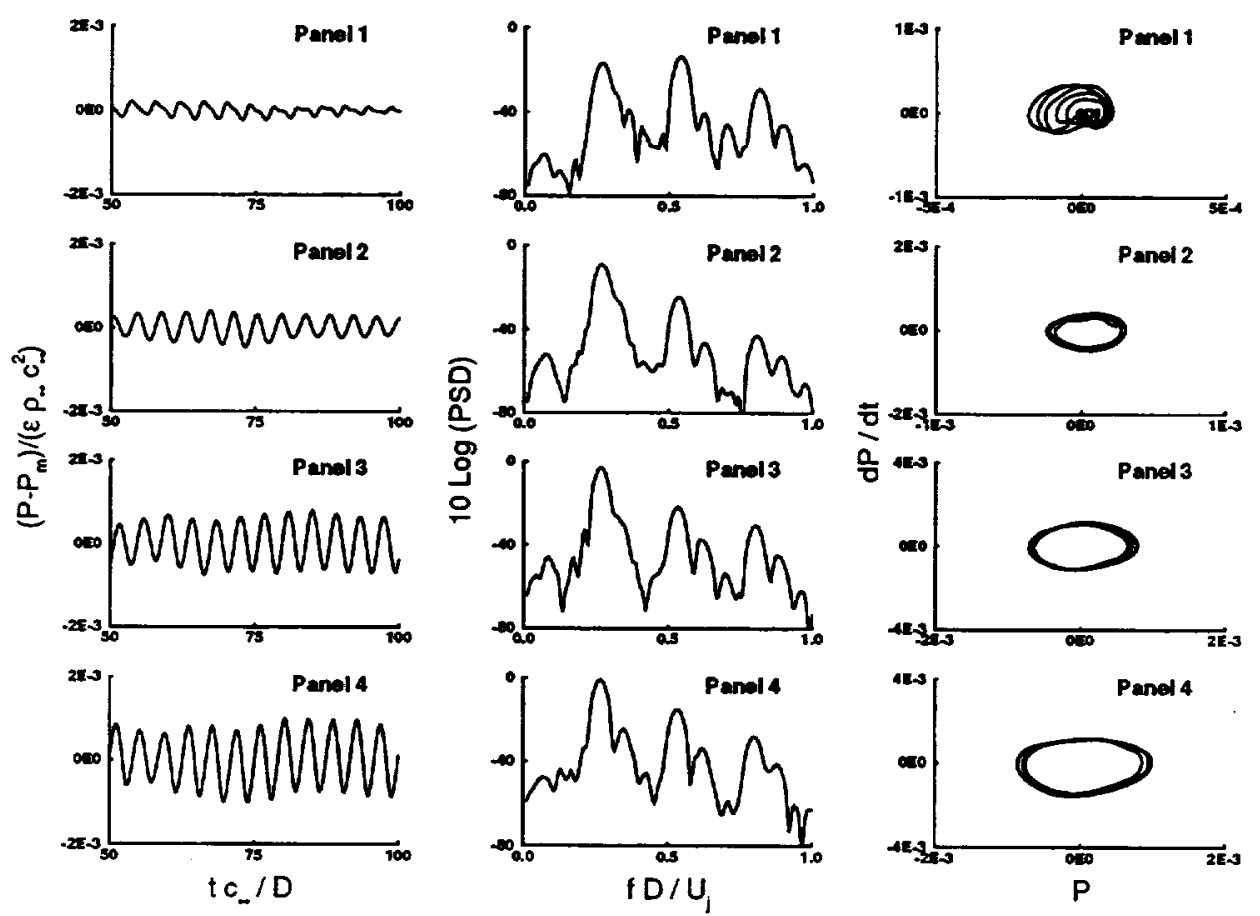

Figure 8: Panel pressure loading time histories, spectra and phase plots 

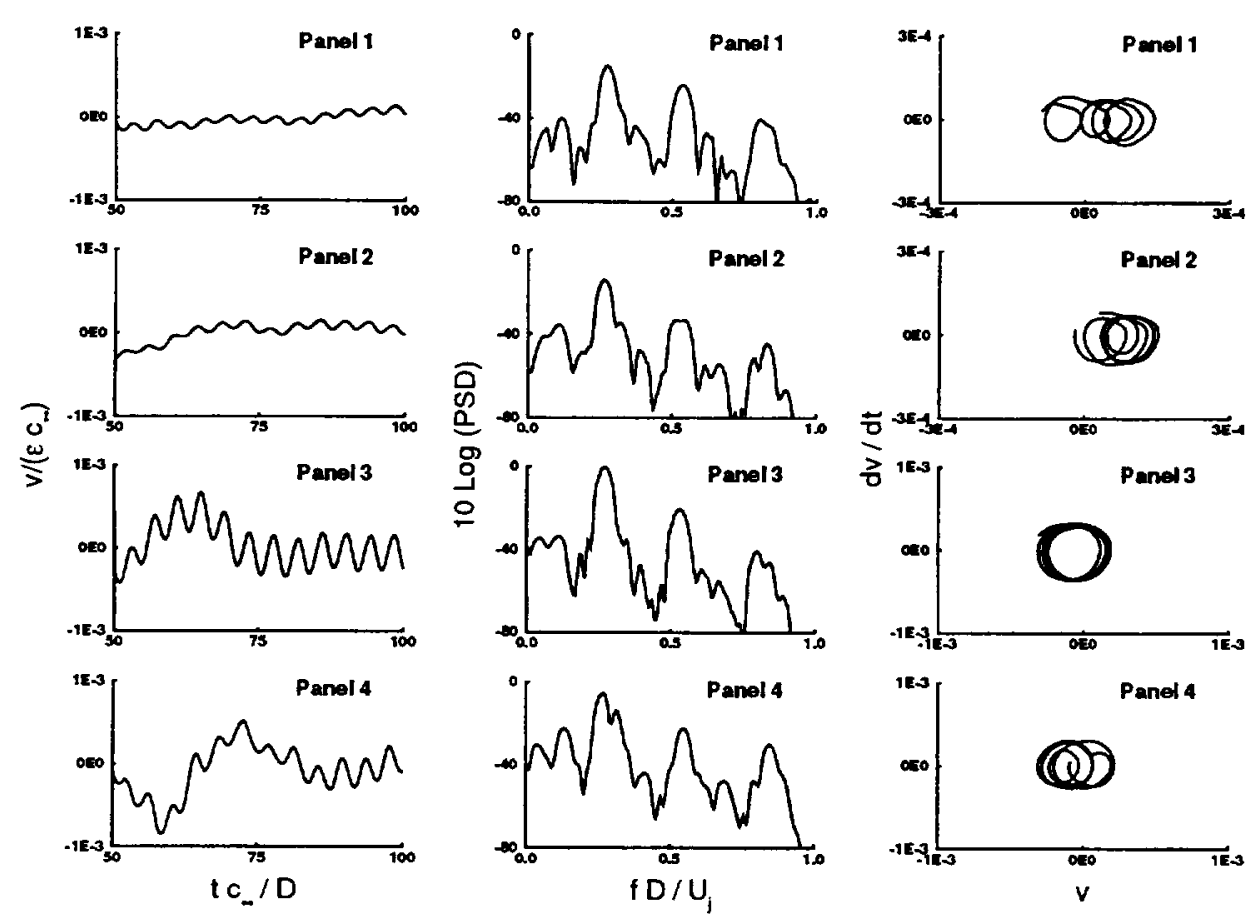

Figure 9: Panel velocity time histories, spectra and phase plots
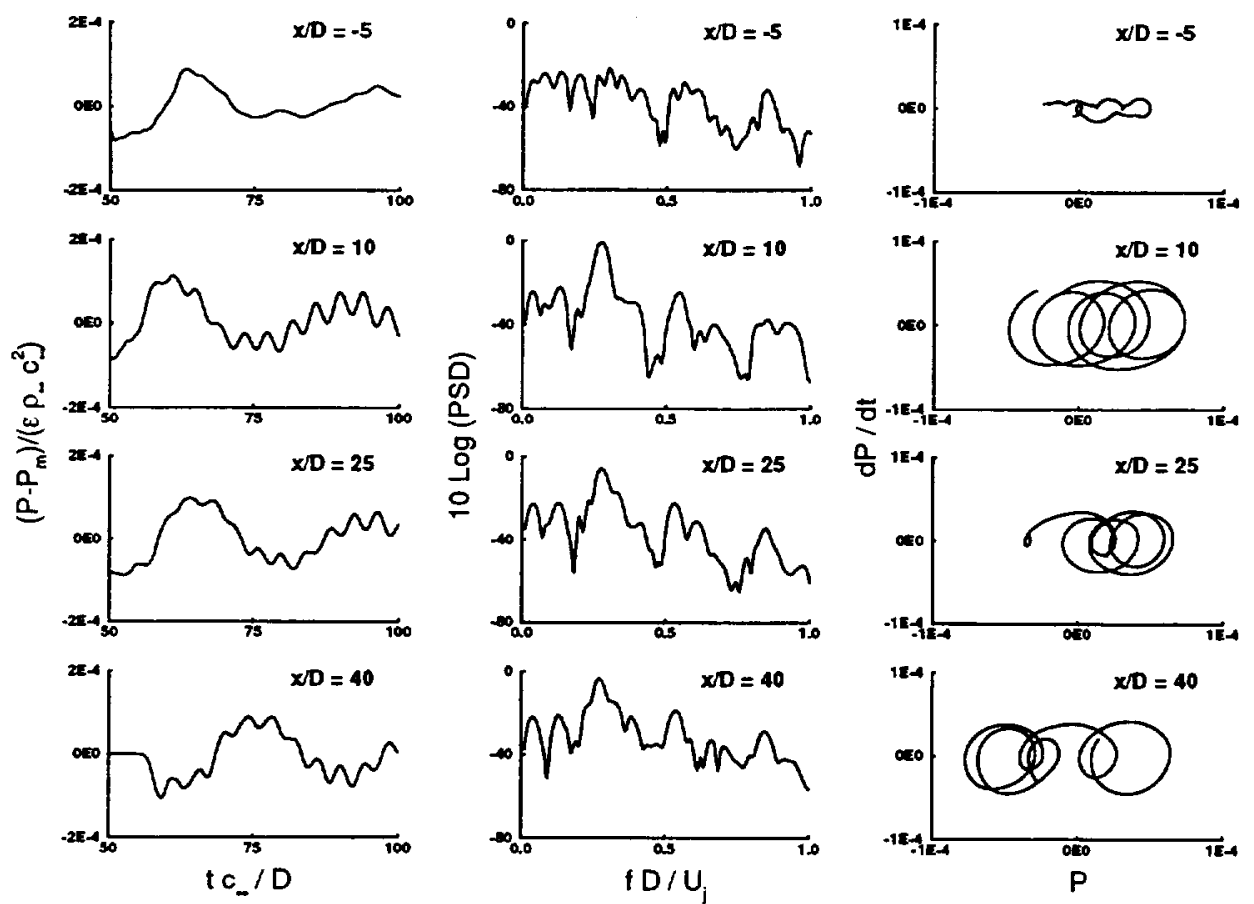

Figure 10: Radiation domain far field time histories, spectra and phase plots at $x / D=-5,10,25$, and 40 along the line $y / D=25$ 


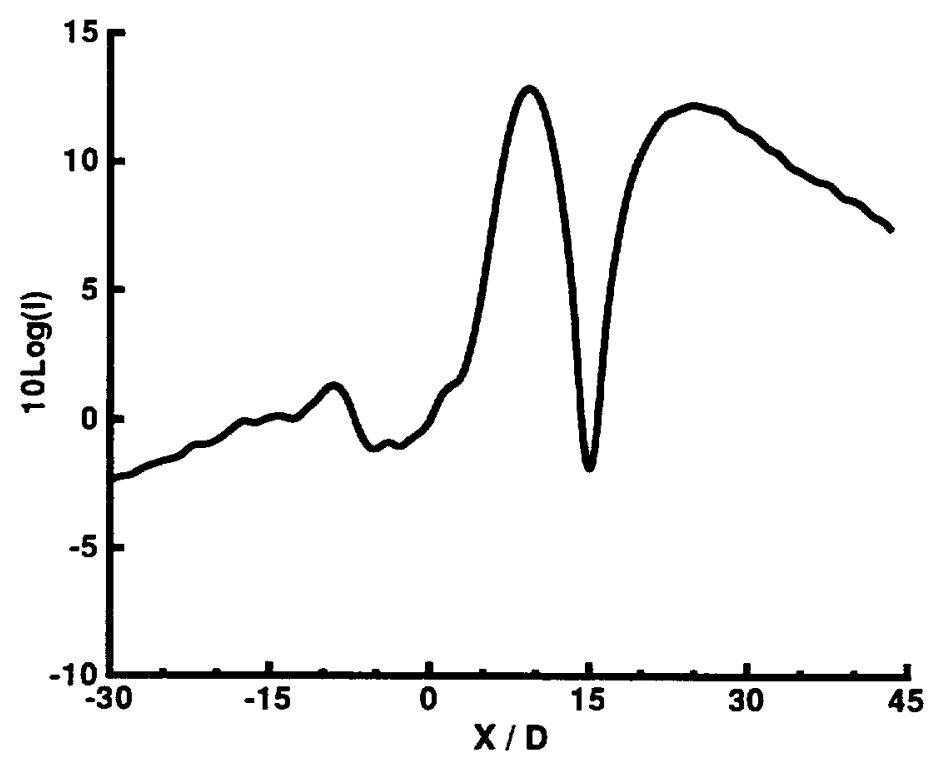

Figure 11: Radiation domain directivity of overall sound pressure level along the line $y=25 D$. 


\begin{tabular}{|c|c|c|}
\hline \multicolumn{2}{|c|}{ REPORT DOCUMENTATION PAGE } & $\begin{array}{l}\text { Form Approved } \\
\text { OMB No. 0704-018s }\end{array}$ \\
\hline \multicolumn{3}{|c|}{ 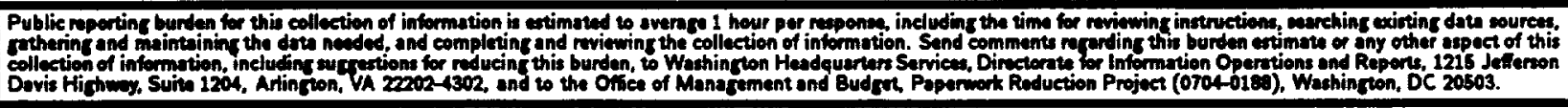 } \\
\hline \multicolumn{3}{|c|}{\begin{tabular}{l|l|l|} 
1. AGENCY USE ONIY(Leave biank) & $\begin{array}{l}\text { 2. REPORT DATE } \\
\text { February } 1996\end{array}$ & $\begin{array}{l}\text { 3. REPORT TYPE AND DATES COVERED } \\
\text { Contractor Report }\end{array}$ \\
\end{tabular}} \\
\hline \multicolumn{2}{|c|}{$\begin{array}{l}\text { 4. TITLE AND SUETITLE } \\
\text { RESPONSE OF A PANEL STRUCTURE FORCED } \\
\text { BY THE NOISE FROM A NEARLY SONIC JET }\end{array}$} & $\begin{array}{l}\text { C NAS1-19480 } \\
\text { WU 505-90-52-01 }\end{array}$ \\
\hline \multicolumn{3}{|l|}{$\begin{array}{l}\text { G. AUTHOR(S) } \\
\text { C. C. Fenno, Jr. } \\
\text { A. Bayjiss } \\
\text { L. Maestrello }\end{array}$} \\
\hline \multicolumn{2}{|c|}{$\begin{array}{l}\text { 7. PERfORMiNG ORGANizaTION MAME(S) AND ADDRESS(ES) } \\
\text { Institute for Compater Applications in Science and Engineering } \\
\text { Mail Stop 132C, NASA Langley Research Center } \\
\text { Hampton, VA 23681-0001 }\end{array}$} & $\begin{array}{l}\text { 8. PERFORMING ORGANIZATION } \\
\text { REPORT NUMBER } \\
\text { ICASE REPOrt No. } 96-11\end{array}$ \\
\hline \multicolumn{2}{|c|}{$\begin{array}{l}\text { 9. SPONSORING/MONITORING AGENCY NAME(S) AND ADORESS(ES) } \\
\text { National Aeronautics and Space Administration } \\
\text { Langley Research Center } \\
\text { Hampton, VA } 23681-0001\end{array}$} & $\begin{array}{l}\text { 10. SPONSORING/MONITORING } \\
\text { AGENCY REPORT NUMBER } \\
\text { NASA CR-198282 } \\
\text { ICASE RePOIt No. } 96-11\end{array}$ \\
\hline \multicolumn{3}{|c|}{$\begin{array}{l}\text { 11. SUPPLEMENTARY NOTES } \\
\text { Langley Technical Monitor: Dennis M. Bushnell } \\
\text { Final Report } \\
\text { Submitted to AIAA Journal }\end{array}$} \\
\hline \multicolumn{2}{|c|}{$\begin{array}{l}\text { 120. DISTRIBUTION/AVAILABILITY STATEMENT } \\
\text { Unclassified-Unlimited } \\
\text { Snbject Category } 34\end{array}$} & 126. DISTRIBUTION CODE \\
\hline \multicolumn{3}{|c|}{$\begin{array}{l}\text { 13. ABSTRACT (Maximum } 200 \text { monds) } \\
\text { A model of a high subsonic jet with a nearby array of flexible, aircraft-type panels is studied numerically in two } \\
\text { dimensions. The jet is excited by a limited duration, spatially localized starter pulse in the potential core. The } \\
\text { long time evolution of unsteady disturbances in the jet, the responses of the panels and the ensuing radiation } \\
\text { are computed. The results show that the spectral response of both the jet and the panels is concentrated in a } \\
\text { relatively narrow frequency band centered at a Strouhal number (based on jet exit velocity) of approximately } 0.25 \\
\text { and associated harmonics. The loading on the panels generally increases with downstream distance. Panel radiation } \\
\text { is weakest in upetream directions. Interior zones of silence, due to destructive interference of radiation from the } \\
\text { panels, are observed. }\end{array}$} \\
\hline
\end{tabular}

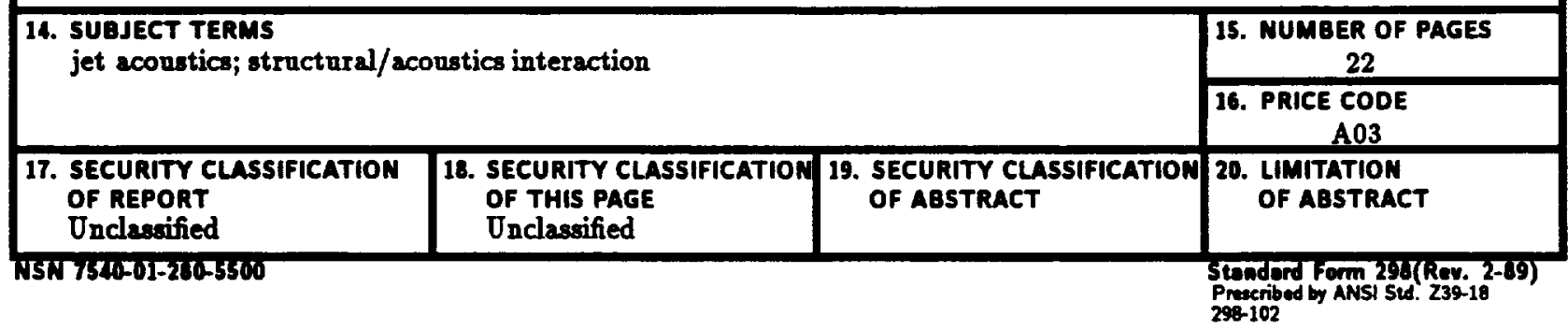



